\title{
Combinatorial Chemistry. Synthesis, Analysis, Screening. Edited by Günther Jung (guenther.jung@uni-tuebingen.de). Wiley-VCH: Weinheim. 1999. XXXII+602 pp. 268 DM. ISBN 3-527-29869-X
}

\section{Shu-Kun Lin}

Molecular Diversity Preservation International (MDPI), Saengergasse 25, CH-4054 Basel, Switzerland Tel.: +41 79322 3379, Fax: +41 61302 8918, E-mail: lin@mdpi.org, URL: http://www.mdpi.org/lin/

Received: 29 December 1999 / Published: 23 February 2000

This is not only a book written by specialists for specialists, but also for everyone who wants to brush up on their knowledge on the rapidly and ever-growing field of combinatorial chemistry. It is an indispensable source of information for researchers working in organic synthesis, catalysis, biochemistry, and biotechnology, pharmaceutical and clinical chemistry, material sciences, and analytical chemistry.

I was glad to see that Professor Ivar Ugi is the main author of a chapter (Chapter 4. Combinatorial Chemistry of Multicomponent Reactions). He has been long regarded by many as the father of combinatorial chemistry because of his initial studies on multicomponent organic reactions.

It is a fact that the main topic of combinatorial chemistry is organic synthesis, and that for obvious reasons, quantitative (100\%) yields are preferred for combinatorial synthesis. Solid-phase synthesis is also preferred. Chemistry is an experimental science. Chemists may find working on synthesis, analysis and all kinds of bioactivity tests to be tedious. If possible, chemists would like to use automatic processes for synthesis, analysis and screening. These may be possible if these complicated processes can be clearly defined as unit operations. Ideally, in future, all unit operations or chemical reactions, separations, characterization and bioactivity tests, etc., can be carried out by individual "modules" which are controlled by computer. Normally high-throughput screening has been regarded as the cause of sudden and extensive exploration in combinatorial chemistry. Expectations for automation might be another driving force. The processes handled by machine are expected to be much different than classical ones; for instance, the size of the container can be much smaller than the flask we hold in our hands when we perform synthetic experiments. We can rely on analytical instruments (probes) which

(C) 2000 by MDPI (http://www.mdpi.org). Reproduction is permitted for noncommercial purposes. 
can be much better than our eyes and noses.

Actually, combinatorial chemistry may be a topic covering both combinatorial synthetic processes and combinatorial structures. Analysis as a step in the process can be inserted anywhere. For those compounds provided as part of compound libraries, the analysis of individual compounds might be necessary. Structural characterization of compounds can be carried out after activity assessments (another kind of analysis) and only for those active compounds.

Screening is duly included as one of the three main topics in this book because many bioactivity tests are simplified to a single ligand-target combination, very much like a bimolecular reaction.

The twenty chapters listed are the following:

1. Combinatorial Chemistry.

2. Survey of Solid Phase Organic Reactions.

3. Solution Phase Combinatorial Chemistry.

4. Combinatorial Chemistry of Multicomponent Reactions.

5. Solid Phase Anchors in Organic Chemistry.

6. The Use of Templates in Combinatorial Chemistry for Solid Phase Synthesis of Multiple Core Structure Libraries.

7. Combinatorial Synthetic Oligomers.

8. Glycopeptide and Oligosaccharide Libraries.

9. RNA and DNA Aptamers.

10. Combinatorial Approaches to Molecular Receptors for Chemosensors.

11. Peptide Libraries in T-Cell Mediated Immune Response.

12. Combinatorial Biosynthesis of Microbial Metabolites.

13. Design and Diversity Analysis of Compound Libraries for Lead Discovery.

14. How to Scale-up While Scaling Down: EVOscreen, a Miniaturized Ultra High Throughput Screening System.

15. High Throughput Experimentation in Catalysis.

16. FT-Infrared Spectroscopy and IR-Microscopy for On Bead Analysis of Solid Phase Synthesis.

17. High-Resolution Magic Angle Spinning NMR Spectroscopy of On-Bead Analysis of Solid Phase Synthesis.

18. Mass Spectrometric Analysis of Organic Compound Collections.

19. Automation in Combinatorial Synthesis.

20. Ion-Cyclotron-Resonance-FT-Mass Spectrometry for Elemental Composition Analysis of Library Components.

Acknowledgments: I am grateful to Günther Jung (guenther.jung@uni-tuebingen.de) and Wiley-VCH's Sonja Karlein (skarlein@wiley-vch.de) for their kind assistance.

(C) 2000 by MDPI (http://www.mdpi.org). Reproduction is permitted for noncommercial purposes. 